\title{
Intuitionistic fuzzy sets and their use in image classification
}

\author{
Alžbeta Michalíková ${ }^{1,2}$ \\ ${ }^{1}$ Faculty of Natural Sciences, Matej Bel University \\ Tajovského 40, Banská Bystrica, Slovakia \\ e-mail: alzbeta.michalikova@umb.sk \\ ${ }^{2}$ Mathematical Institute, Slovak Academy of Sciences \\ Ďumbierska 1, Banská Bystrica, Slovakia
}

Received: 31 January 2019

Accepted: 9 April 2019

\begin{abstract}
In this paper, the problem of classification of images is discussed. Our specific problem is that we need to classify tire images into selected classes which are characterized by some patterns. The theory of intuitionistic fuzzy sets is used for classification of the images. In the first step is showed the way how this type of images could be represented as the vectors. Then the membership and non-membership value to each coordinate are calculated and finally the value of similarity measure between patterns and specific image is computed. Classification is performed with respect to the valued of similarity measure.
\end{abstract}

Keywords: Intuitionistic fuzzy sets, Similarity measure, Image classification.

2010 Mathematics Subject Classification: 03C98, 03E72.

\section{Introduction}

Intuitionistic fuzzy sets (shortly IFSs) were introduced by Krassimir Atanassov in 1983 [1]. Since then, many new properties and applications of this mathematical structure have been constructed. In this paper, we will use IFSs for image classification of the pictures of tires. The motivation for this application is an ongoing cooperation between the Department of Computer Science of Matej Bel University and the local criminal police department and the recognition and classification of 
the tire prints is one of the basic problems being addressed. In the paper we will show one of the first steps of this process, namely, obtaining the samples which could be used as elements of a database for comparing with the tire printd found at the crime scene. The pictures of tires are obtained from the internet and then they are automatically processed by application developed by us.

The paper is structured as follows: In the Section 2 we give a brief introduction into the theory of intuitionistic fuzzy sets and we define the properties which are used in this paper. In the Section 3 we discuss the way how we could prepare the data for classification. In Section 4 the obtained results are summarized and finally in the Section 5 the conclusions and some ideas for future work are mentioned.

\section{Intuitionistic fuzzy sets}

Definition 1. Let $X$ be a universe. An intuitionistic fuzzy set is a set

$$
A=\left\{\left\langle x, \mu_{A}(x), \nu_{A}(x)\right\rangle \mid x \in X\right\}
$$

of the functions $\mu_{A}: X \rightarrow[0,1], \nu_{A}: X \rightarrow[0,1]$ such that

$$
0 \leq \mu_{A}(x)+\nu_{A}(x) \leq 1 .
$$

Function $\mu_{A}$ is called the membership function and function $\nu_{A}$ is called the non-membership function.

By $\mathcal{F}$ we will denote the family of all IFSs. There exists another one function defined on $\mathcal{F}$, function $\pi_{A}$ which is defined as

$$
\pi_{A}(x)=1-\mu_{A}(x)-\nu_{A}(x) .
$$

This function is called hesitation margin.

Let us have two intuitionistic fuzzy sets $A=\left(\mu_{A}, \nu_{A}\right), B=\left(\mu_{B}, \nu_{B}\right)$. Then it holds

$$
\begin{aligned}
& A=B \Longleftrightarrow \mu_{A}=\mu_{B} \& \nu_{A}=\nu_{B} \\
& A \leq B \Longleftrightarrow \mu_{A} \leq \mu_{B} \& \nu_{A} \geq \nu_{B}
\end{aligned}
$$

Definition 2. Let $S$ be a real-valued function such that $S: \mathcal{F} \times \mathcal{F} \rightarrow[0,1]$. $S$ is called a similarity measure if for every $A, B, C \in \mathcal{F}$ it holds

- $S(A, B)=S(B, A)$,

- $S(A, B)=1$ iff $A=B$,

- If $A \leq B \leq C$ then $S(A, C) \leq S(A, B)$ and $S(A, C) \leq S(B, C)$.

In this paper we consider the discrete universe $X=\left\{x_{1}, x_{2}, \ldots, x_{h}\right\}$ and for classification we use the cosine-base similarity measure

$$
S_{C}(A, B)=\frac{1}{h} \sum_{i=1}^{h} \frac{\mu_{A}\left(x_{i}\right) \mu_{B}\left(x_{i}\right)+\nu_{A}\left(x_{i}\right) \nu_{B}\left(x_{i}\right)}{\sqrt{\mu_{A}^{2}\left(x_{i}\right)+\nu_{A}^{2}\left(x_{i}\right)} \sqrt{\mu_{B}^{2}\left(x_{i}\right)+\nu_{B}^{2}\left(x_{i}\right)}} .
$$

This IFSs similarity measure has been designed by Ye [6]. 


\section{Preparation of the data}

As it was mentioned in the Introduction, we are using images which are downloaded from the internet. The basic idea is to download each image (together with its model identifier) which is on the website with the name of tire brand. This process is described in the paper [4]. Then we have a large set of different images. In Figure 1, there are shown the most often occurring types of images.

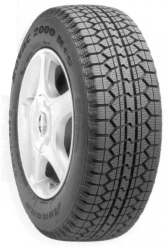

(a) Class 1

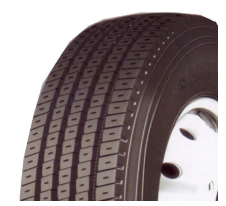

(b) Class 2

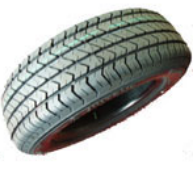

(c) Class 3

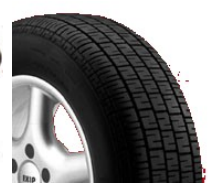

(d) Class 4

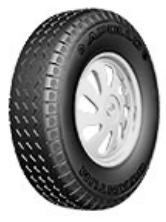

(e) Class 5

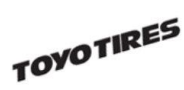

(f) Class 6

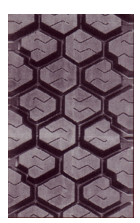

(g) Class 7

Figure 1. Seven types of tire images

We are focused on those images where the tire prints are best visible and therefore the most interesting for us are those with whole wheels (Figures 1a, 1e) and those with tire print (Figure $1 \mathrm{~g})$. The classification of the set of images into the seven classes will be presented in this paper. The next procedure with the data is to find the ellipses which make a boundaries of tire print (see $[3,5]$ ), take the rectangle with sample of print and after some modification adding it into our database (see Figure 2). This database is created for comparing actually known tire prints with print found in the crime scene.
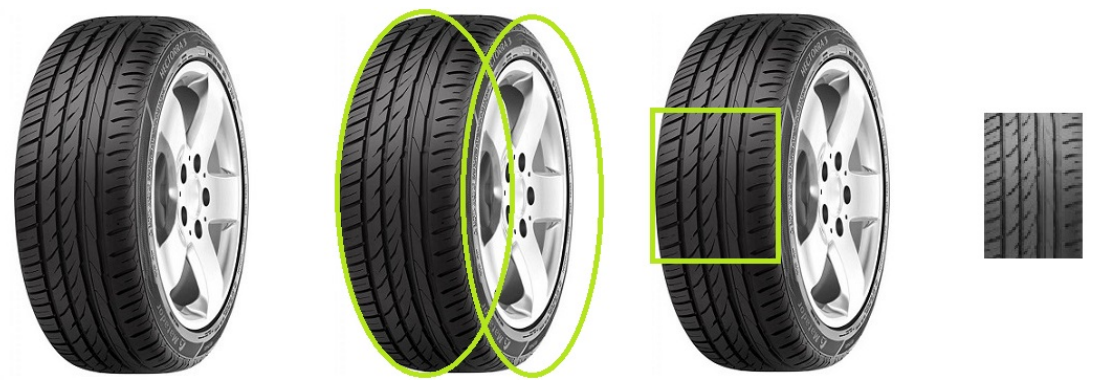

Figure 2. Procedure of preparing the data into the database

\subsection{Preprocessing of the images}

From each image we want to extract as much information as possible, therefore, we do not resize the images into the same size. Our preprocessing consists of the following steps:

1. Converting of the image into the graphical format .jpg,

2. Removing of the white pixels on the all sides of the image, 
3. Converting of the image into the black and white colour,

4. Dividing of the image into the 16 parts (Figure 3).

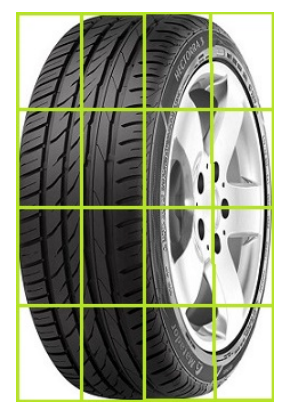

Figure 3. Dividing of the image

All the algorithms are programming in the program MATLAB. We decide to divide the image into the 16 parts because for the classification we would like to use vector representation of the images. Our idea is that if we look at the Figure 1, we can see that for example image on the Figure 1a has in the left upper corner approximately half of the pixels white and half of the pixels black. On the other side the image on the Figure 1c has most of the pixels in the left upper corner white and for example the image on the Figure 1d has in this corner most of the pixels black.

As it was mentioned already, we will represent each image by the vector with 16 coordinates where each coordinate represent the number of white pixels in given part. Since we have images of the different sizes first we calculate the values of matrix $M$ of size $4 \times 4$ by the formula

$$
M_{k, l}=\frac{16 B_{k, l}}{x y}
$$

where values $x, y$ represent the original size of the image and $B_{k, l}$ represent the number of white pixels in the part with order $k$ and $l$. After this calculation we simply change the matrix $M$ into the vector $V$.

\subsection{Preprocessing of the data}

In this part the way how to assign the degree of membership and the degree non-membership to each image vector coordinate is described. First, we work with the set of the templates. As the templates we take 3 images of each group of tires which are displayed on the Figure 1. So we have 21 templates which are represented as vectors. For preprocessing of the data we use the approach that was described in the paper [2]. Let us have image $i(i=1,2, \ldots, 21)$ represented by a 16-coordinates vector

$$
V_{i}=\left(v_{i, 1}, v_{i, 2}, \ldots, v_{i, 16}\right) .
$$

We start with the normalization of each coordinate by using formula

$$
n_{i, j}=\frac{v_{i, j}-\bar{X}_{j}}{s_{j}},
$$


where $j=1,2, \ldots, 16, \bar{X}_{j}$ is a mean and $s_{j}$ is the standard deviation calculated from the $j$-th coordinate of all images in the template database. Then the membership degree of each template coordinate is calculated by the weighted sigmoid function

$$
\mu_{i, j}=\frac{r_{j}}{1+e^{-n_{i, j}}}
$$

where $r_{j}$ is a weight value that is computed as

$$
r_{j}=\left|\frac{1-s_{j}}{1+s_{j}}\right| .
$$

Similarly, the non-membership degree of each template coordinate is calculated by the formula

$$
\nu_{i, j}=\frac{r_{j}}{1+e^{n_{i, j}}} .
$$

Since we are dividing the images into the seven classes we define the seven patterns by the formula

$$
P_{m}=\left\{\left\langle\bar{v}_{m, j}, \bar{\mu}_{m, j}, \bar{\nu}_{m, j}\right\rangle\right\}_{j=1}^{16},
$$

where $m=1,2, \ldots, 7$ and the values $\bar{\mu}_{m, j}$ and $\bar{\nu}_{m, j}$ represent the arithmetical mean of function values of those templates which belong to given class.

\subsection{Classification of the images}

Now we are ready to classify any image. We are using following algorithm:

1. Take any image,

2. Use preprocessing and characterize image by 16-coordinates vector (see Section 3.1),

3. For each coordinate calculate its membership and non-membership value by the same formulas like they were used for templates (see Section 3.2),

4. Classify image into the suitable class by using the similarity measure $S_{C}$.

To classify the image into the suitable class, we calculate the value of similarity measure $S_{C}$ between each pattern $P_{m}(m=1,2, \ldots, 7)$ and given image. The image is classified into that class where the value of similarity measure is the highest.

\section{Experimental results and discussion}

For this experiment we took 326 images which were downloaded from the web with the different names of tire brand. We developed a software program that preprocesses the images by using the mentioned methods. As a result, the program creates seven folders and moves the images into the folders as they were classified by the described process. There was also given one template image into the each new folder. This could help us quickly identify the incorrectly classified images. We noticed that some of the incorrectly classified images have quite similar values of two best 
values of similarity measure. On the other hand, the difference between the best and the second best similarity value is more different if the image is classified into the right class. Therefore, we decide to create also a text file in which we extract the information about the difference between the best and the second best similarity value.

\begin{tabular}{|c||c|c|c|}
\hline Class & Correct & Incorrect & Sum \\
\hline \hline Class 1 & 40 & 5 & 45 \\
Class 2 & 34 & 2 & 36 \\
Class 3 & 8 & 0 & 8 \\
Class 4 & 48 & 5 & 53 \\
Class 5 & 123 & 6 & 129 \\
Class 6 & 7 & 6 & 13 \\
Class 7 & 36 & 6 & 42 \\
\hline
\end{tabular}

Table 1. Result of classification

The result of classification is displayed in the Table 1. From these data we could calculate that $90.8 \%$ of images were classified correctly. If we look at the incorrectly classified images, we could find some common properties of them. The difference between best and second best similarity value for correctly classified images is almost always greater than the value $0.01(86.2 \%)$. For incorrectly classified images, this value reached the value in less than 0.01 in $36.7 \%$ of cases. If some correctly classified image reached the value of the difference between best and second best similarity value in less than 0.01 , then this image is usually the image, which a human also finds hard to classify exactly into the one class. For example, the image on Figure 4a was classified into the Class 1 but image on Figure 4b was classified into the Class 4 . The next example is the image on the Figure $4 \mathrm{c}$ which was classified into the Class 7.

Another problem is if there is too much captured light on the image. For example, image on Figure $4 \mathrm{~d}$ was classified into the Class 1 but it actually belongs to the Class 5 .

The worst results reach the classification of the text (Class 6). Into this class were again assigned the images with much captured light, for example image on Figure 4e.

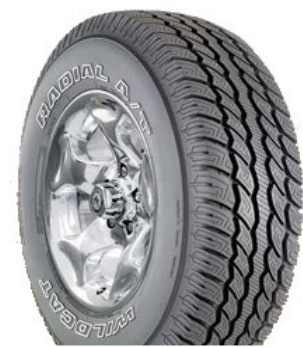

(a)

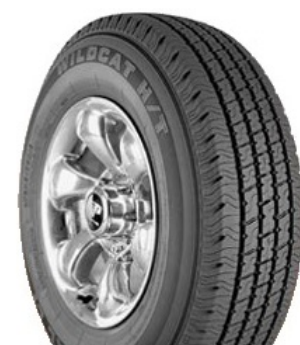

(b)

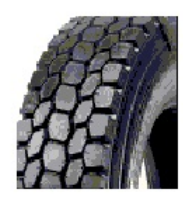

(c)

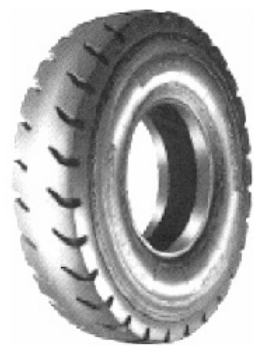

(d)

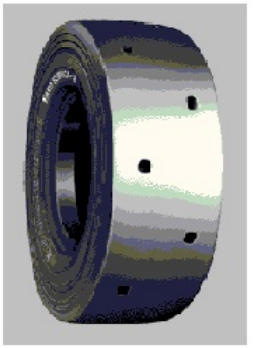

(e)

Figure 4. Images mentioned in discussion 


\section{Conclusions}

In this paper, we use intuitionistic fuzzy sets for the classification of the images of tires. We describe the way how the image of a car tire could be represented by a vector. Then for each vector we determine the value of membership and also the value of non-membership function. We use the cosine similarity measure to calculate the similarity of the image with the predetermined patterns. We classify the set of images and discuss the problems of incorrect classification. The main advantage of this approach is that it has a high percentage of success and it can be used in automated processing of the images which are obtaining from the web. In the future, we would like to try to use other similarity measures. For example, those similarity measures which use not only the values of membership and non-membership function but they take into the account also the value of hesitation margin. We will try to verify if it is possible to improve our results by using this function.

\section{Acknowledgements}

The support of the grant KEGA 025UMB-4/2017 is kindly announced.

\section{References}

[1] Atanassov, K. T. (1983). Intuitionistic fuzzy sets, VII ITKR Session, Sofia, 20-23 June 1983 (Deposed in Centr. Sci.-Techn. Library of the Bulg. Acad. of Sci., 1697/84) (in Bulgarian). Reprinted: Int. J. Bioautomation, 2016, 20(S1), S1S6.

[2] Intarapaiboon, P. (2016). Text classification using similarity measures on intuitionistic fuzzy sets. SCIENCEASIA, 42 (1), 52-60.

[3] Michalíková, A. \& Vagač, M. (2016). A tire tread pattern detection based on fuzzy logic. Flexible Query Answering Systems 2015. Springer, Cham, 381-388.

[4] Vagač, M., Melicherčík, M., Marko, M., Trhan, P., Michalíková, A., Kliment R. \& Drapka, R. (2015). Crawling images with web browser support. 13th International IEEE Scientific Conference on Informatics'2015, 286-289.

[5] Vagač, M., Melicherčík, M. \& Schon, J. (2015). Classification of tire images in order to obtain the best possible tire tread sample. The 5th International Scientific Conference, Applied Natural Science 2015. September 30-October 2, 2015, Jasn; Trnava : UCM, 173.

[6] Ye, J. (2011). Cosine similarity measures for intuitionistic fuzzy sets and their applications. Mathematical and Computer Modelling, 53(1-2), 91-97.

[7] Zadeh, L. A. (1965). Fuzzy sets. Information and Control, 8 (3), 338-353. 